"This is the peer reviewed version of the following article: [Petrovska $K$, Watts NP, Catling C, Bisits A, Homer CS. Supporting Women Planning a Vaginal Breech Birth: An International Survey. Birth 22 Aug 2016], which has been published in final form at [Link to final article using the DOI]. This article may be used for non-commercial purposes in accordance with Wiley Terms and Conditions for Self-Archiving." 
Petrovska K, Watts NP, Catling C, Bisits A, Homer CS. Supporting Women Planning a Vaginal

Breech Birth: An International Survey. Birth 22 Aug 2016. DOI: 10.1111/birt.12249

\section{Supporting women planning a vaginal breech birth: An international survey}

\section{Abstract}

Objective: The aim of this study was to explore the experiences of women who planned a vaginal breech birth.

Method: An online survey was developed consisting of questions regarding women's experiences surrounding planned vaginal breech birth. The survey was distributed between April 2014 - January 2015 to closed membership Facebook groups that had a consumer focus on vaginal breech birth.

Results: There were 204 unique responses to the survey from women who had sought the option of a vaginal breech birth in a previous pregnancy. Most women (80.8\%) stated they were happy with the birth choices they made and a significant proportion (89.4\%) would attempt a vaginal breech birth in subsequent pregnancies. Less than half of women were formally referred to a clinician skilled in vaginal breech birth when their baby was diagnosed breech (41.8\%), while the remainder sourced a clinician themselves. Half of the women felt supported by their care provider (56.7\%) and less than half (42.3\%) felt supported by family and friends.

Conclusion: The women who responded to this international survey sought the option of a vaginal breech birth, were subsequently happy with this decision and would attempt a vaginal 
breech birth in their next pregnancy. Access to vaginal breech birth is important for some women, however this choice may be challenging to achieve. Consistent information and support from clinicians is important to assist decision-making.

Key words: decision-making, information, support, vaginal breech birth. 


\section{Background and Objective}

Breech presentation is estimated to occur in 3-5\% of pregnant women at, or close to, term [1]. Most breech presenting babies are now born by elective caesarean section. Data on the percentage of women with breech babies who seek planned vaginal birth is limited, however in Australia, 2013 data shows that of the 13, 617 babies who were diagnosed breech at term (4\% of all babies born), $88 \%$ were born by caesarean section [2]. The remaining $12 \%$ of vaginal breech births in Australia in 2013 included both planned and unplanned births, suggesting that figures for planned vaginal breech birth are much fewer. This trend is echoed across a number of high income countries $[3,4]$. The low number of vaginal breech births is largely attributed to an international randomised control trial published in 2000, known as the Term Breech Trial, which concluded that caesarean section was the safest mode of birth for babies in the breech position [5]. Subsequent research and systematic reviews have also been released reinforcing the Trial's results $[6,7]$, with one study noting that vaginal breech birth carries a two to five fold greater relative risk of short term morbidity and mortality than caesarean section [4] .

Since the Term Breech Trial was published, many have challenged the methods and findings of this research [8-11]. Subsequent research has also concluded that vaginal breech birth can be a safe option with the appropriate care and expertise for carefully selected women [11-14]. However, many maternity services across the world have been reluctant to respond to this evidence, with the number of clinicians skilled in vaginal breech birth decreasing to almost nonexistent levels [11]. This limits the birth options available to pregnant women with breech 
presenting babies and results in most women, in many regions across the world, having a planned caesarean section [13].

Despite the limited support for vaginal breech birth in maternity facilities, some women continue to request it as an option [15]. Women who plan a vaginal breech birth are often highly motivated and value autonomy in their decision making for birth. Previous studies have indicated that these women undertake their own research and choose to navigate the health system to find a clinician who is willing to assist them to give birth vaginally $[11,15,16]$.

The limited amount of quality information about vaginal breech birth and the importance of responding to consumer demand for access to vaginal breech birth was recently highlighted in The Lancet [3]. Therefore, this study was undertaken to explore the experiences of women who reported choosing a vaginal breech birth and were motivated to seek supportive care and information that assisted them to access this option for birth. This study also aimed to increase understanding in how to best support these women and provide quality information.

\section{Methods}

This study was initially prompted by a qualitative research project that interviewed women on their decision-making for vaginal breech birth [15]. In order to further investigate women's decision-making, a survey was developed to explore experiences nationally and internationally.

The electronic survey was developed by the research team for distribution to women online via social media. The survey focussed on women's expectations and experiences surrounding planned vaginal breech birth, including methods used by the woman to source a clinician skilled 
in vaginal breech birth and the level of support and quality of information provided from clinicians. The survey consisted of five and ten point Likert scale questions as well as open ended questions for participants to add further information if desired. Ethical approval for distribution of the survey was granted by the relevant local Human Research Ethics Committee.

The survey was piloted with two volunteers who had planned a vaginal breech birth; one had a vaginal birth, the other a caesarean section in labour. The survey was modified following feedback that focused on the need to ensure instructions were clear and unambiguous. As a result, some questions were split to distinguish between clinician 'helpfulness' and clinician 'influence'.

The survey was uploaded onto SurveyMonkey ${ }^{\circledR}$, an online platform that allows the distribution of research surveys for a nominal fee. Once uploaded, the survey was distributed via closed membership Facebook groups from the United States, United Kingdom and Australia that had a focus on vaginal breech birth. The survey was posted online from April 2014 to January 2015. Two reminders were posted during that period to reach women who may not have seen previous posts about the survey.

Women who were interviewed for the original research conducted on their decision-making experiences for vaginal breech birth [15] were also given the opportunity to complete the survey. It is unclear as to how many of these women responded given the anonymity of the survey submissions.

All potential respondents were provided with clear information about the nature of the data being collected and the identity of the organisation holding the data. They were also advised of 
the purpose for which the data were going to be used and advised that all responses were anonymous. The survey took approximately 30 minutes to complete.

Data from the Likert Scales were analysed using descriptive statistics. Responses to questions were optional and the number of respondents for each question varied. Therefore, the denominator varied depending on the number of responses. The Likert scale questions were grouped into 3 categories (Agree, Neutral and Disagree) for analysis and reporting purposes. Data from the open ended questions included in the survey was significant in volume and will be analysed using thematic analysis that will be reported elsewhere.

\section{Results}

In total 204 women who had previously planned a vaginal breech birth responded to the survey. A significant proportion were from the United States (36.2\%) or Australia (29.7\%). Many of the participants (44.3\%) were between 31-35 years of age. More than three-quarters of the women had tertiary education (76.3\%). Over one third of those who responded to the question relating to parity $(62.9 \%)$ had given birth more than once (Table 1$)$.

When asked about referral to a clinician skilled in vaginal breech birth, less than half of women who responded to this question (41.8\%) were referred to, and subsequently saw, a clinician who was skilled in vaginal breech birth when their baby was noted to be in the breech presentation. Women not referred to a skilled clinician sourced one independently. About half (54.2\%) of the women who saw a skilled clinician received information at the first meeting to assist them in their decision-making. Of these women, almost half (49.4\%) were satisfied with 
the information they received while a third (33.8\%) were dissatisfied. Of the 164 women who disclosed their mode of birth, 104 women had a vaginal breech birth (63.4\%).

Women were asked about the usefulness of being provided with detailed statistics on the safety and risk of vaginal breech birth in helping them make a decision regarding mode of birth. Over half (53.9\%) agreed that the statistics were useful, while one quarter (24.4\%) felt undecided.

The helpfulness of the information from clinicians was explored. Two-thirds $(66.7 \%)$ of the women agreed that information received from a midwife was helpful in choosing the way they wanted to give birth while one fifth (20.3\%) were unsure. About one-third $(32.9 \%)$ of these women agreed that information received from a doctor was helpful in choosing the way they wanted to give birth, while almost half $(49.3 \%)$ disagreed.

Access to the internet for information on vaginal breech birth was investigated. Three quarters of respondents (74.5\%) felt that the information they found on the internet was helpful for choosing the way they wanted to give birth. Less than half of these women (43.1\%) felt the information they found online was from reliable sources, and a third (33.3\%) felt that information online had an influence on their birth choices.

Women were asked about the major external influences on their decision-making in the survey. Among those that responded, almost half (49.7\%) agreed that their midwife had influenced their decision-making regarding mode of birth, while almost a third (30.5\%) indicated that their doctor had an influence. Family and friends had the least influence, with less than a quarter of these women (21.3\%) agreeing that family and friends had an impact on their birth choices. 
Almost half (42.0\%) felt that family and friends held no influence over their decisions regarding their birth.

Women were asked about the support they received during decision making for birth. Just over half $(56.7 \%)$ felt supported by their care provider while one-quarter $(22.7 \%)$ remained unsure. Less than half of these women (42.3\%) agreed that they felt supported by family and friends. Almost all (85.8\%) agreed that they would have liked to have spoken to other women who had been through the same experience.

Despite varying levels of satisfaction with information and support from care providers and family and friends, most women (89.4\%) stated that they would attempt a vaginal breech birth in a subsequent pregnancy if their baby presented in the breech position and most $(80.8 \%)$ were happy with the decision made for the birth of their baby.

\section{Discussion}

Our study aimed to explore women's experiences of planning a vaginal breech birth. The findings of this survey showed that levels of satisfaction with care and support varied, however many of the women who responded indicated that they were happy with the choice they made and would attempt a vaginal breech birth in a subsequent pregnancy. Our study suggests that access to vaginal breech birth was important for these women.

In our previous qualitative study, we found that the women interviewed wanted the option for a vaginal breech birth as it was considered essential to have a choice in their mode of birth [15]. They saw 'having a go' at vaginal birth as a 'rite of passage' and a primal test of womanhood. 
The results of the quantitative data in this study support the notion that access to skilled clinicians to explore the opportunity to give birth to their baby vaginally is important.

This is demonstrated in the findings related to the impact of external influences on the decision making process, with a significant proportion of women either disagreeing or remaining neutral regarding external factors influencing their choices. Although midwives were noted to have a stronger influence than doctors in the decision to try for a vaginal breech birth, these findings note that overall, many of the respondents found that the clinicians they came into contact with did not have a strong influence on their decision to pursue a vaginal breech birth. Similarly, the internet and family influences were not seen as major influences.

Most women would have liked to have spoken to other women during this period, indicating that they felt a sense of isolation during this time. This is supported in our qualitative study of women's decision-making for vaginal breech birth [15], as well as other research [1], that suggests women feel alone in the process of making decisions about mode of birth for breech presentation.

Gaining access to helpful information was a common challenge for women who participated in this study. The challenge for clinicians is to provide this information using an unbiased, nonjudgmental approach, which may be difficult given that many maternity clinicians today are heavily influenced by current practices and medico-legal contexts relating to the option of vaginal breech birth $[12,15]$. Communicating management options for breech presentations may be related to local attitudes regarding vaginal breech birth and historical contexts following the findings of the Term Breech Trial and other studies. The Term Breech Trial 
changed practice in many clinical settings across the world, effectively reducing or removing the option for vaginal breech birth for many women [4]. Countries that continued to offer the option of vaginal breech birth, despite the findings of the Term Breech Trial retained clinician skill and have higher numbers of vaginal breech births than those that were significantly more affected by the Trial's findings $[8,11,15,17]$. Maintenance of clinician skill and general acceptance of vaginal breech birth as a viable option for birth may therefore account for increased satisfaction from women regarding clinician support for their options [15].

Despite reporting dissatisfaction with information received from clinicians and the limited influence on their decision-making, two thirds of the women felt supported by their care providers. It was unclear if the care providers referred to in this question were the same as those in previous questions where they reported dissatisfaction with information and levels of influence. It may suggest that, for some women, a change in care provider to a clinician who was more supportive of vaginal breech birth occurred. This was consistent with results from our previous qualitative study of women seeking a vaginal breech birth in New South Wales, Australia [15]. Most women in that study had to fight against the health system for the option of vaginal birth and were met with clinicians who they felt used 'scare tactics' to highlight negative consequences of vaginal breech birth as a means to dissuade them. The majority of these women were initially not in a hospital supportive of vaginal breech birth which meant finding a skilled clinician and transferring to another hospital (one that supported vaginal breech birth) or to another clinician within the same facility. 
Less than half of women in the survey felt supported by family and friends, suggesting that most women's immediate social circle had negative attitudes towards vaginal breech birth. The lack of trust from broader society in the birthing process is not a new concept in the literature [18-22] including studies that focus on women's decision-making processes for breech birth. Findings from a Swiss study demonstrated that the women experienced decisional conflict regarding their birth options [1]. The major factors influencing this conflict were lack of support, social pressures and lack of information. Improved support from clinicians was required and more accurate information should be made available so that women felt more supported during the decision-making process. It also demonstrates the importance of clinicians engaging with women's families and/or significant others when sharing information and discussing the options available.

Our survey was available online and in English only. It may have been useful to have elicited views from non-English speaking countries that may have a more liberal policy toward vaginal breech birth. The potential for sampling bias may have impacted on the results, as only women motivated for a vaginal breech birth and who were members of closed Facebook groups were likely to have responded. This self-selected convenience sample were also women likely to have been passionate about birth choices, which is reflected in the results indicating that many of the women would attempt a vaginal birth for a breech baby in a subsequent pregnancy. Therefore, this sample may not be representative of all women who have a breech baby or choose the option of vaginal breech birth. Responses to questions were optional and the number of respondents for each question varied, which may have impacted on the conclusions drawn from the findings. Despite these considerations, this is the first international study to 
explore women's experiences of vaginal breech birth and the support they received in deciding on mode of birth. The findings support the need for larger studies that explore women's experiences when choosing vaginal breech birth.

\section{Conclusion}

The women who responded to this international survey sought the option of a vaginal breech birth, were subsequently happy with this decision and would attempt a vaginal breech birth in their next pregnancy. Many of the women in this study appeared to receive varying degrees of information and support for the option of vaginal breech birth. Access to vaginal breech birth is important for some women, however this choice may be challenging to achieve. Consistent information and support from clinicians is important to assist decision-making and planning care in the antenatal period. 


\section{References}

1. Guittier, M.-J., et al., Breech presentation and choice of mode of childbirth: A qualitative study of women's experiences. Midwifery, 2011. 27(6): p. e208-e213.

2. AlHW, Australia's mothers and babies 2013-in brief. 2015, Australian Institute of Health and Welfare: Canberra.

3. van Roosmalen, J. and T. Meguid, The dilemma of vaginal breech delivery worldwide. The Lancet, 2014. 383(9932): p. 1863-1864.

4. Berhan, Y. and A. Haileamlak, The risks of planned vaginal breech delivery versus planned caesarean section for term breech birth: a meta-analysis including observational studies. BJOG: An International Journal of Obstetrics \& Gynaecology, 2016. 123(1): p. 49-57.

5. Hannah, M.E., et al., Planned caesarean section versus planned vaginal birth for breech presentation at term: a randomised multicentre trial. The Lancet, 2000. 356(9239): p. 13751383.

6. Hofmeyr, G.J., M. Hannah, and T.A. Lawrie, Planned caesarean section for term breech delivery. Cochrane Database of Systematic Reviews, 2015(7).

7. Vlemmix, F., et al., Term breech deliveries in the Netherlands: did the increased cesarean rate affect neonatal outcome? A population-based cohort study. Acta Obstetricia et Gynecologica Scandinavica, 2014. 93(9): p. 888-896.

8. Glezerman, M., Five years to the term breech trial: The rise and fall of a randomized controlled trial. American Journal of Obstetrics and Gynecology, 2006. 194(1): p. 20-25.

9. Kotaska, A., Inappropriate use of randomized trails to evaluate complex phenomena: Case study of vaginal breech delivery, in British Medical Journal. 2004. p. 1039 - 1042.

10. Hauth, J.C. and F.G. Cunningham, Vaginal breech delivery is still justified. Obstetrics \& Gynecology, 2002. 99(6): p. 1115-1116.

11. Lawson, G.W., The Term Breech Trial Ten Years On: Primum Non Nocere? Birth, 2012. 39(1): p. 3-9.

12. Glezerman, M., Planned vaginal breech delivery: current status and the need to reconsider. Expert Review of Obstetrics \& Gynecology, 2012. 7(2): p. 159-166.

13. Kotaska, A., et al., SOGC clinical practice guideline: Vaginal delivery of breech presentation: no. 226, June 2009. Int J Gynaecol Obstet, 2009. 107(2): p. 169-76.

14. Goffinet, F., et al., Is planned vaginal delivery for breech presentation at term still an option? Results of an observational prospective survey in France and Belgium. American Journal of Obstetrics \& Gynecology, 2006. 194(4): p. 1002-1011.

15. Homer, C.S.E.e.a., Women's experiences of planning a vaginal breech birth in Australia. BMC pregnancy and childbirth, 2015. 15: p. 89.

16. Ecker, K., A Breech in the System, K. Ecker, Editor. 2009, Freestyle Art Films: Australia. p. p. 43 minutes. Byron Bay, Australia.

17. Glas $\emptyset$, A.H., I.M. Sandstad, and E. Vanky, Breech delivery - what influences on the mother's choice? Acta Obstetricia et Gynecologica Scandinavica, 2013. 92(9): p. 1057-1062.

18. Fisher, C., Y. Hauck, and J. Fenwick, How social context impacts on women's fears of childbirth: A Western Australian example. Social Science \& Medicine, 2006. 63(1): p. 64-75.

19. Reiger, K. and R. Dempsey, Performing birth in a culture of fear: an embodied crisis of late modernity. Health Sociology Review, 2006. 15(4): p. 364-373.

20. Murphy, M.A., Medicalization of birth: The social construction of Cesarean section: $A$ qualitative analysis. 2010, University of Michigan: Ann Arbor. p. 187. 
21. West, J.E., Birth Matters: Discourses of Childbirth in Contemporary American Culture, in English. 2011, Louisiana State University: Baton Rouge. p. 254.

22. Davis-Floyd, R., Birth as an American rite of passage. 2003, Berkely: University of California Press. 
Table 1: Women's experiences planning a vaginal breech birth April 2014 to January 2015 Women's demographic characteristics

\begin{tabular}{|c|c|}
\hline Variable & n (\%) \\
\hline Country of origin & $n=185$ \\
\hline United States & $67(36.2)$ \\
\hline Australia & $55(29.7)$ \\
\hline United Kingdom & $22(11.9)$ \\
\hline Canada & $17(9.1)$ \\
\hline New Zealand & $14(7.5)$ \\
\hline South Africa & $3(1.6)$ \\
\hline Germany & $2(1.0)$ \\
\hline Other & $5 \quad(2.7)$ \\
\hline Age (years) & $n=203$ \\
\hline $18-25$ & $9(4.4)$ \\
\hline $26-30$ & $47(23.2)$ \\
\hline $31-35$ & $90(44.3)$ \\
\hline $36-40$ & $38(18.7)$ \\
\hline$>41$ & $19(9.4)$ \\
\hline $\begin{array}{l}\text { No. of children (including } \\
\text { index pregnancy) }\end{array}$ & $n=197$ \\
\hline 1 & $73(37.1)$ \\
\hline 2 & $64(32.5)$ \\
\hline
\end{tabular}




\begin{tabular}{|l|c|}
\hline 3 & $28(14.2)$ \\
\hline 4 & $19(9.6)$ \\
\hline 5 & $13(6.6)$ \\
\hline Education & $\mathbf{n = 1 9 0}$ \\
\hline High School & $31(16.3)$ \\
\hline University & $145(76.3)$ \\
\hline Apprentice/Technical & $16(8.4)$ \\
\hline Mode of birth & $\mathbf{n = 1 6 4}$ \\
\hline Vaginal & $104(63.4)$ \\
\hline Em. CS & $60(36.6)$ \\
\hline
\end{tabular}

